und Biguanidverbindungen mittelst Natronkalks hat der Verfasser in Uebereinstimmnng mit Makris (Lieb. Ann. 184, 371) die Verbrennung des entstehenden Ammoniaks erkannt. Ein weiterer, aber kaum nennenswerther Verlust ist dadurch bedingt, dass sich nach Vollendung der Analyse Spuren von Cyansäure im Natronkalk vorfinden.

Reclamation die Bestimmung des in den Methylalkoholen enthaltenen Acetora betreffend, von Vincent und Delachanal (Bull. soc. chim. [3], 3, 681-682).

Ein selbstregulirender Gasgenerator nach neuem Principe, von H. W. Hillger (Amer. Chem. Journ. 12, 228-230). Zum Verständnisse des Apparates ist die Hilfe der Zeichnung nöthig.

\title{
Bericht über Patente
}

von

\section{Ulrich Sachse.}

Berlin, den 11. Juni 1890.

Metalle. Salzbergwerk Neu-Stassfurt in Loederburg bei Stassfurt. Retorten ofen zur Behandlung vonpulverförmigen Substanzen mit Gasen bei Glühhitze. (D. P. 51209 rom 12. Mai 1889 , Kl. 40.) Der Ofen besteht aus mehreren stehenden Retorten, in welchen entweder durch Auskleidung mittelst feuerbeständigen Materials Bänke oder darch Einmauerung von besonders geformten Steinen in den Retortenwandungen Vorsprünge gebildet werden. Auch kann man in Absätzen mauern, um dadurch jene Bänke herzustellen. Durch diese innere Einrichtung der Retorten soll erreicht werden, dass die letzteren sich nicht verstopfen, wodurch sonst das Durchstreichen der Gase gehindert würde.

J. C. von Breinlstein in Wien. Apparat zur Gewinnung der metallhaltigen Theile aus metallführendem Gestein oder Sand. (D. P. 50929 rom 14. August 1889, Kl. 1.) Behufs Abscheidung specifisch schwererer von specifisch leichteren 
Substanzen sind ein Quirlapparat und eine Centrifuge derart mit einander combinirt, dass die zu behandelnde erz-oder metallhaltige Trübe zuerst durch den Quirlapparat und die aus letzterem austretende, des grössten Theiles der specifisch schwereren Substanz beraubte Trübe durch die Centrifuge geht. Wird dieser Apparat zur Goldgewinnung benutzt, so empfiehlt es sich, in die Centrifuge zur Erhöhung der Leistungsfäbigkeit eine angemessene Menge Quecksilber einzufübren. In Betreff der Ausführung des Apparates muss auf die Patentschrift verwiesen werden.

R. E. Moffatt in New-York, V. St. A. Verfahren und Einrichtungen zum Trennen von Metallen aus Gemengen unter Anwendung von Elektromagneten, welche durch Wechselströme erregt werden. (D. P. 50930 vom 17. September 1889, KI. 1.) Das Gemenge passirt ein Magnetfeld, dessen Kraftlinien in Folge der Anwendung von Wechselströmen beständig wechseln. In Folge dessen sollen die nicht magnetischen Theilchen abgelenkt und die magnetischen angezogen und beide in besondere Behälter befördert werden.

S. W. Cragg aus Baltimore, Maryland, V. St. A., z. Z. in Paris. Verfahren zur Gewinnung von Gold und Silberaus edelmetallhaltigen Erzen mittelst trockenen Chlorgases. (D. P. 51117 vom 21. Juni 1889, Kl. 40.) Die Erzmassen werden mit trockenem Chlorgas in einem mit Dampfmantel umgebenen Gefässe in der Weise behandelt, dass durch den einströmenden Dampf oder ein anderes gasförmiges Heizmittel die Temperatur in dem Chlorirungsgefäss während der Einwirkung des Chlorgases $100-150^{\prime \prime}$ beträgt. Die zu chlorirenden Erzmassen und die Chlorgase kommen mit dem Erwärmungsmittel nicht in Berührung. Auf diese Weise soll der Chlorirungsprocess sehr beschleunigt werden. Das Auslaugen der Chlormetalle geschieht in hölzernen Gefässen, die mit einem Asphaltubberzug, Doppelboden und Filter versehen sind.

R. C. Thompson in St. Helens, Junction Lane, Lancaster, England. Verfahren und Apparat zur Gewinnung von Zinn, Loth und dergleichen aus Weissblechabfällen. (D. P. 50735 von 27. Juni 1889, Kl. 40.) Behufs Gewinnung von Zinn, Loth und dergl. aus Weissblechabfällen werden letztere bis über den Schmelzpunkt der Ueberzugs-Metalle und Metalllegirungen unter Verwendung von solchen Gasen (Wasserstoff, Stickstoff ete.) und Flüssigkeiten erhitzt, welche keinen oxydirenden Einfluss auf die za gewinnenden Metalle ausüben. Als Flüssigkeiten, deren Siedepunet höher liegen muss als der Schmelzpunkt des Zinns, will Erfinder Palmöl und das 
als Cylinderöl« bekannte Petroleum benutzen. Diese Stoffe gelangen durch die mit Perforationen versehene hohle Welle in eine mit Schüttelvorrichtung versehene perforirte Trommel, die zur Aufnahme der Abfälle dient. Die zur Erhitzung dienenden Gase und Flüssigkeiten entweichen durch eine obere Oeffnung des die perforirte Trommel ungebenden Mantels, während das abgeschmolzene Zinn durch eine untere Oeffnung desselben Mantels abfliesst.

E. Walsh jr. in St. Louis, Missouri, V. St. A. Apparat zum Condensiren von Zinkdämpfen und Sammeln metallischen Zinks. (D. P. 51208 vom 1. Mai 1889, Kl. 40.) Die Condensationskammer dient gleichzeitig als Beschickungskammer für das kohlenstoffhaltige Material. In letzterem verdichten sich die Zinkdämpfe und das flüssige Metall sammelt sich am Boden der schräg gelagerten Condensationskammer, von wo es durch Röhren nach einem Sammelbehälter abgeführt wird.

A. K. Reinfeld in Wien. Verfahren zur Herstellung leicht abhebbarer Metallniederschläge auf galvanoplastischem Wege. (D. P. 50890 vom 22. November 1888, Kl. 48.) Die Druckplatte oder eine beliebige geeignete Form wird zuerst mit einem Nickelüberzuge versehen, oder man verwendet mit Nickel legirte Platten, da Nickel nur in geringem Maasse die Eigenschaft besitźt, galvanoplastische Niederschläge festzuhalten. Unterwirft man die Nickeloberfäche überdies einer Behandlung mit oxydirenden Körpern (Chromaten, Manganaten) oder seifenartigen Mischungen (Fett - und Harzsäuren in Verbindung mit alkalischen Körpern) zum Zwecke, dieselbe von vertieften Stellen zu befreien und vollständig glatt zu machen, so rerliert sie die genannte Eigenschaft vollkommen, und es wird möglich, leicht abhebbare Metallniederschläge von nur 1-2 Tausendstel Millimeter Dicke herzustellen.

Th. H. Martin in Swansea, England. Bewegliche und zerlegbare Ingotformen zur Fabrikation von Platten, Blechen und sonstigen Gegenständen aus Kupfer and seinen Legirungen. (D. P. 50715 vom 20. October 1888, Kl. 40.) Die Formen werden um Zapfen drehbar oder auseinandernehmbar in besondern Gestellen, die fahrbar eingerichtet werden können, angeordnet. Sie sind mit einem Kupferboden oder Klappboden versehen, nach dessen Lösung die Blöcke (Ingots) direct aus den Formen herausfallen. Derartige Formen sollen dazu dienen, bei der Fabrikation von Platten, Blechen und sonstigen Gegenständen aus Kupfer und seinen Legirungen das sehnelle Herüberschaffen der Stücke von einem Ende der Walze nach dem andern zu ermöglichen. 
Glas. G. Kersten in Firma Boeck \& Kersten, Glasbütte in Charlottenburg bei Berlin. Neuerung in dem Verfahren und den Einrichtungen zur Herstellung von gepressten Glaswaaren. (D. P. 50634 vom 22. November 1888, Kl. 32.) Das in einem einzigen Eingussbehälter eingebrachte flüssige Glas wird durch eine oder mehrere kleine konische Oeffnungen in die Formräume eingedrückt.

M. W. Griswold in Ridgewood, Staat New-Yersey und W. B. Atterbury in Brooklyn, Staat New-York, V. St. A. Verfahren zum A usflachen von Fensterglascylindern. (D. P. 51358 vom 4. September 1889 , KI. 32.) Sobald der aufgerchlitzte Glascylinder im Ofen weich und dehnbar wird, wird derselbe dadurch flach gelegt, dass man einen Strom heisser Luft, heissen Dampfes oder Gases hineinleitet, unter dessen Druck die durch das Aufschneiden erbaltenen Kanten nach aussen nachgeben und der Cylinder sich in eine Fläche ausrollt.

Thonwaaren. A. Schöfer in Lägerdorf bei Itzehoe. Schachtofen für ununterbrochenen Betrieb. (D. P. 50711 vom 14. Mai 1889, KI. 80.) Durch besondere, seitlich vom Brennraum des Schachtofens angebrachte Canäle wird in den Brennraum das Brennmaterial continuirlich zugeführt, während der Schachtofen durch den eigentlichen Schacht in gleicher Weise mit Kalkstein, Cement oder anderem zu brennenden Material beschickt wird.

J. Craven und B. Craven in Wakefield, York, England. Vorrichtung an Ziegelmaschinen, um die Steine zwischen Formtisch und Presse zu wenden. (D.P. 51163 rom 28. Juni 1889, Kl. 80.) Ein zwischen Formtisch und Presse angeordneter Wender bringt die Unterseite des Ziegels automatisch nach oben.

P. Windiseb in Meissen, Sacbsen. Maschine zum Anschneiden von Kacheln, Fliesen u. dergl. vor dem Brennen. (D. P. 51236 vom 10. August 1889, Kl. 80.)

Plastische Massen. J. R. France in New-York, V. St. A. Neuerung im Verfahren zum Entwässern von nitrirter Cellulose und anden daza benutzten hydraulischen Pressen bei der Fabrikation von Cellulö̈d. (D. P. 50921 vom 4. Dezember 1888, Kl. 39.) Dureh das Verfahren soll besonders breiartige Nitrocellulosemasse in grossen Mengen ohne Explosionsgefahr entwässert werden. Dieselbe wird in mehreren Lagen zwischen Zeugstücken aufgeschichtet, wozu geeigneten Falles noch Lagen von Kampher und Farbstoff treten, in bydraulischen Pressen möglichst 
stark abgepresst, worauf zur Entfernung des durch Druck allein nicht entfernbaren Wassers comprimirte Luft, dann Alkohol und schliesslich wieder Luft zur Austreibung des Alkohols hindurchgepresst wird. $\mathrm{Zu}$ diesem $Z_{\text {wecke }}$ ist die hydraulische Presse mit einem besonderen Presskasten verbunden. Derselbe ist zum Auseinandernebmen eingerichtet und mit einem Kopfstück mit starker Siebscheibe und Fussstück versehen, sowie ferner mit einem an den gegenüber liegenden Kanten aus einander zu nehmenden Behälter mit auswechselbarem falschen Boden. Luft und Alkohol treten oben durch die Siebscheibe des Kopfstückes in den Pressstoss ein und unten durch den falschen Boden wieder aus.

Färberei, Appretur u. s. w. H. Erdmann in Halle a./S. Verfahren zum Färben von Haaren und Federn. (D. P. 51073 vom 6. Juli 1889. Zisatz zum Patent $47349^{1}$ ) vom 26. October 1888, KI. 8.) Anstatt der im Hauptpatent genannten Diamine werden $\gamma$-Amidophenol, 8 -Triamidophenol, $1=5$-Dioxynaphtalin (Schmelzpunkt des Acetats $160^{\circ}$ ) oder Mischungen dieser Körper zum Braun-oder Schwarzfärben von Kopf- und Barthaar des Menschen angewandt und zwar entweder in Combination mit Oxydationsmitteln (auch der Luft) oder mit Chinon und Toluchinon, welche Körper sich gleicherweise auch mit den Diaminen des Hauptpatentes zu braunen bis schwarzen Furbstoffen auf Haaren und Federn combiniren lassen.

E. Friedlein in Würzburg. Verfahren zur Herstellung von Maltuch. (D. P. 51111 vom 7. Mai 1889, Kl. 8.) Völlig ebenes Maltuch wird dadurch hergestellt, dass man Leinwand in gespanntem Zustand auf eine polirte Platte aus Cement, Marmor, Glas oder dergl. legt und von der Rückseite aus mit einer gelatinösen, die Leinwand durchziehenden Masse übertränkt. Diese Masse wird aus Leimlösung, einer Emulsion von Ricinusöl, arabischem Gummi und Farbstoff, wie Zink-, Blei- oder Barytweiss zusammengemischt.

Organische Verbindungen, verschiedene. A. Artmann in Braunschweig. Verfahren zur Herstellung von Sulfosäuren beziehungsweise deren Salzen aus von Pheuolen befreiten Theerkohlenwasserstoffen zu Desinfectionszwecken (D. P. 51515 vom 1. Mai 1889, Kl. 12.) Die von Phenolen befreiten Theerkohlenwasserstoffe werden sulfurirt und die in wässrige Lösung gebrachten Sulfosäuren durch Aussalzen abgeschieden. Neutralisirt und mit Theerkohlenwasserstoffen vermischt, sollen diese Säuren als Desinfectionsmittel Verwendung finden.

1) Diese Berichte XXII, 3, 363. 
B. Philips in Aachen und L. Diehl in Genf. Verfahren zur Darstellung von Fehling'sche Lösung nicht reducirenden Acetylderivaten des unsymmetrischen Methyl-oder unsymmetrischen Aethylphenylhydrazins. (D. P. 51597 vom 16. März 1889, Kl. 12.) Das Verfahren bezweckt die Darstellung von Fehling'sche Lösung nicht reducirenden Monoäthylverbindungen unsymmetrischer secundärer Hydrazine, speciell des unsymmetrischen Aethylphenylhydrazins und des unsymmetrischen Methylphenylhydrazins. Wie bekannt, sind diese Basen von E. Fischer ${ }^{1}$ ) zuerst dargestellt worden, lassen sich aber auch nach A. Michaelis ${ }^{2}$ ) aus Natriumphenylhydrazin erhalten; praktische Verwerthung wird für den rorliegenden Fall wohl nur die Methode von E. Fischer (Reduction des aus dem Monomethyl- oder Monoäthylanilin gewonnenen Nitrosamins) finden. Die Acetylirung des auf diese Weise gewonnenen unsymmetrischen Aethyl- oder Metbylphenylhydrazins kann durch Einwirkung von Essigzäureanhydrid auf die Base oder durch Kochen derselben mit Essigsäure mit oder ohne Zusatz eines Condensationsmittels, wie entwässertes essigsaures Natron erfolgen.

Beispiel: 10 Gewichtstheile unsymmetrischen Aethylphenylhydrazins werden allmählich unter Umrühren mit 8 Gewichtstheilen Essigsäureanhydrid versetzt. Nach einiger Zeit wird die ganze Masse fest; durch Umkrystallisiren aus Wasser oder verdünntem Alkohol wird das Reactionsproduct rein erhalten. Dasselbe bildet farblose, geschmack- und geruchlose Krystalle, welche bei $87^{\circ}$ (uncorr.) schmelzen und in Wasser, Alkohol, Aether und Ligroin löslich sind. Die Bildung des Körpers erfolgt nach der Gleichung:

$\mathrm{C}_{6} \mathrm{H}_{5}>\mathrm{N} . \mathrm{NH}_{2}+\left(\mathrm{C}_{2} \mathrm{H}_{3} \mathrm{O}\right)_{2} \mathrm{O}=\mathrm{C}_{6} \mathrm{C}_{2} \mathrm{H}_{5}>\mathrm{N} . \mathrm{NH} \cdot \mathrm{C}_{2} \mathrm{H}_{3} \mathrm{O}+\mathrm{C}_{2} \mathrm{H}_{4} \mathrm{O}_{2}$. Acetyläthylphenylhydrazin.

Dasselbe Product wird erbalten durch Einwirkung von Acetylchlorid auf unsymmetrisches Aethylphenylhydrazin. Ein analoges Product lässt sich nach den oben beschriebenen Methoden aus unsymmetrischem Methylphenylhydrazin und Essigsäureanhydrid beziehungsweise Eisessig oder Acetylchlorid darstellen. Die so erhaltenen Körper (speciell das beschriebene Acetyläthylphenylbydrazin) sollen technische und medicinische Verwendung finden.

Seifen und Fette. W. Rödiger, in Firma Kluge \& Co., in Magdeburg. Neuerung in der Darstellung harter Harzseifen. (D. P. 50817 vom 14. November 1888. Zusatz zum Patent

1) Ann. Chem. Pharm. 190, 152 und diese Berichte VIII, 1642.

2) Diese Berichte XIX, 2448. 
45960') vom 5. April 1888, Kl. 23.) Der Zusatz des wasserfreien Natriumcarbonats zur Harzseife beim Verfahren des Hauptpatents wird so regulirt, dass sich ein hartes Hydrat des harzsauren Natriums bilden kann, indem man entweder die Bindung des überschüssigen Wassers durch das zugesetzte wasserfreie Natriumcarbonat mittelst Erhöhung der Temperatur bis auf $80^{\circ}$ oder durch Zusatz von etwa $5 \mathrm{pCt}$. Chlornatrium oder durch beide Mittel befördert, oder, falls in der Harzseife weiches, wasserarmes, harzsaures Natrium vorbanden ist, die Bildung des Hydrats durch Zusatz von Wasser oder Krystallsoda in auszuprobirender Menge herbeiführt. Dieses Verfahren wird auch bei der Fabrikation von Harzfettseifen angewandt, deren an Natron gebundene Säuren zu mehr als $50 \mathrm{pCt}$. aus Harzsäuren bestehen.

A. Lommatzsch in Lindenau und J. Herzog in PlagwitzLeipzig. Neuerung bei der Entfettung von Putzwolle u. dergl. in Centrifugal-A pparaten. (D. P. 50989 vom 3. Mai 1889, Kl. 23.) Das Entfettungsmittel (Benzin oder Schwefelkohlenstoff) lässt man auf die Putzwolle in dampfförmigem Zustande in der Centrifuge während gleichzeitiger Einwirkung der Centrifugalkraft einwirken, um möglichst wenig Entfettungsmittel zu gebrauchen. Zu dem $Z$ wecke sind in die in einem geschlossenen Kasten rotirende Centrifuge Vertheilungsrohre durch Stopfbüchsen dampfdicht eingeführt und zwar sind die Rohre entweder nur theilweise beweglich und werden durch eine Schubstange mittelst Excenters zur Vertheilung des Dampfes des Lösungsmittels auf- und niederbewegt, oder sie sind vollständig in der Stopf büchse beweglich und dann mit mehreren Dampfausströmungsrohren versehen. Der Benzindampf wird aus einem besonderen Erzenger zugeleitet and der überschüssige Benzindampf in einer Kühlschlange condensirt. Die Putzwolle liegt in der Centrifuge zwischen zwei concentrischen Cylindersieben; sie wird von den Benzindümpfen unter theilweiser Condensation durehtränkt, so dass das Oel oder Fett dünnflüssig wird und abgeschleudert werden kann.

Tabak. R. Reiter in Göppingen. Cigarren und Cigaretten mit unverbrennbaren Hüllen. (D. P. 51416 vom 19. Juli 1889, Kl. 79.) Der zu den Hüllen verwendete Stoff besteht aus Asbestfasern, welche wie bei der Papierfabrikation verarbeitet werden. Die beim Ranchen nicht mit verbrennende Asbesthülle dient der Reinlichkeit wegen nur zum einmaligen Gebrauch und, nachdem die Cigarre oder Cigarette aufgeraucht ist, dazu, dem Zerstreuen der Asche vorzubeugen. Ausserdem gestattet sie, den zurückbleibenden Ueberrest bequem und ohne Beschmutzung and Gefährdung der etwa damit in Berührung kommenden Gegenstände fortzulegen.

1) Diese Berichte XXII, 3, 155. 
Zucker. C. Adant in Brüssel. Neuerung an Centrifugen mit abnehmbarer Lauftrommel zur Herstellung von Zuckerscheiben für die Würfelzuckerfabrikation. (D. P. $50955 \mathrm{vom}$ 2. Juni 1889; Zusatz zum Patent 44660 vom 1. Januar 1888, Kl. 89.)

Büttner \& Meyer in Uerdingen a./Rh. Apparatzum Behandeln von abzupressenden Zuckerrüben-Schnitzeln mit Kalkmilch. (D. P. 50990 vom 28. Mai 1889, KI. 89.) Mittelst dieses Apparates sollen ausgelaugte Rüben- oder Zuckerrohr-Schnitzel mit Kalkmilch selbstthätig in der Weise imprägnirt werden, dass die aufgenommene Menge Kalk procentisch stets gleich bleibt. Der Apparat besteht aus einem Behälter mit einer Transportschnecke, welche in geneigter Lage theilweise in die im Behälter enthaltene Kalkmilch eintaucht und in einem Troge aus Siebmaterial liegt. Die Schnitzel treten durch eine Schüttvorrichtung am untersten Punkte der Transportschnecke ein und werden, während sie von derselben emporgehoben und gewendet werden, von der Kalkmilch durchströmt. Ein in dem Behälter rotirendes Flügelrad hält die Kalkmilch in beständiger Circulation. Die in der Schnecke gleichzeitig mit gehobene Kalkmilch fliesst durch eine Rinne in den Behälter zurück. 